Quim. Nova, Vol. 25, No. 2, 246-253, 2002.

\title{
EMPREGO DE PLANEJAMENTO FATORIAL PARA A OTIMIZAÇÃo DAS TEMPERATURAS DE PIRÓLISE E ATOMIZAÇÃo DE Al, Cd, Mo E Pb POR ETAAS
}

\author{
Edenir R. Pereira-Filho, Ronei J. Poppi e Marco A. Z. Arruda* \\ Departamento de Química Analítica, Instituto de Química, Universidade Estadual de Campinas, CP 6154, 13083-970
}

Campinas - SP

Recebido em 11/1/01; aceito em 25/7/01

\begin{abstract}
EMPLOYMENT OF FACTORIAL DESIGN FOR OPTIMIZATION OF PIROLISYS AND ATOMIZATION TEMPERATURES FOR Al, Cd, Mo AND Pb DETERMINATION BY ETAAS. This work describes a factorial design for the optimization of pyrolysis and atomization temperatures in ETAAS. As examples, $\mathrm{Cd}$ and $\mathrm{Pb}$ were determined using lower pyrolysis and atomization temperatures and $\mathrm{Al}$ and Mo with higher pyrolysis and atomization temperatures. Good results were obtained for Cd employing $\mathrm{Rh}\left(\mathrm{m}_{\mathrm{o}}=1.4 \mathrm{pg}\right)$ as a permanent modifier with pyrolysis and atomization temperatures of 640 and $1500{ }^{\circ} \mathrm{C}$, respectively. For $\mathrm{Zr}$, $\mathrm{W}$ or $\mathrm{Zr}+\mathrm{W}$, the $\mathrm{Cd}$ pyrolysis and atomization temperatures were 500 and $1500{ }^{\circ} \mathrm{C}$, respectively, with $\mathrm{m}_{\mathrm{o}}=1.4 \mathrm{pg}$ using $\mathrm{Zr}$ or W and $1.5 \mathrm{pg}$ using $\mathrm{Zr}+\mathrm{W}$. The best results for $\mathrm{Pb}$ were those using $\mathrm{Rh}, \mathrm{Zr}, \mathrm{W}$ and $\mathrm{Zr}+\mathrm{Rh}$, obtaining characteristic masses of 42, 37, 34 and $36 \mathrm{pg}$, respectively. Pyrolysis and atomization temperatures of 910 and $1850{ }^{\circ} \mathrm{C}$, respectively, were achieved for this metal. For $\mathrm{Al}$, the best results were obtained when $\mathrm{Zr}$ or $\mathrm{Zr}+\mathrm{W}$ were used. Mo was also tested as a possible permanent modifier for Al, but the results were not satisfactory. The results obtained for Mo without modifier were similar to those with conventional modifiers $(\mathrm{Mg}$ or $\mathrm{Pd}+\mathrm{Mg}$ ) and the results obtained using permanent chemical modifiers were not satisfactory. In all situations, the experiments were performed faster than those using the univariate procedure.
\end{abstract}

Keywords: factorial design; permanent chemical modifier; metals.

\section{INTRODUÇÃO}

Atualmente a possibilidade de obtenção de uma grande quantidade de dados numéricos tem crescido em todos os campos da ciência, incluindo a química analítica, devido ao desenvolvimento de novas técnicas e instrumentação que permitem uma resposta de forma mais rápida ${ }^{1}$. Neste contexto, a aplicação de ferramentas estatísticas é de fundamental importância, principalmente para explorar e entender uma gama crescente de dados e informações originadas de um sistema ${ }^{2}$.

O planejamento fatorial ${ }^{3-5}$ é uma ferramenta estatística importante e simples, porém, pouco empregada ou explorada em espectrometria atômica. A observação dos efeitos de variáveis e interações entre elas, é de extrema importância para entender os processos que estão sendo monitorados em um determinado sistema. Quando é empregada uma otimização univariada, não é possível detectar interações entre as variáveis estudadas ${ }^{6}$. Na otimização das temperaturas de pirólise e atomização em espectrometria de absorção atômica com atomização eletrotérmica (ETAAS), é comum, inicialmente, fixarmos a temperatura de pirólise, variando-se somente a temperatura de atomização. Em seguida, depois de encontrar a melhor temperatura de pirólise, a temperatura de atomização é variada. Neste tipo de procedimento, alguns resultados e interpretações incompletas podem ocorrer, devido ao fato de que os efeitos de interação entre as variáveis não são explorados ${ }^{6}$. Além do problema relatado anteriormente, o número de experimentos executados é geralmente maior se comparado com àqueles obtidos em um planejamento fatorial ${ }^{4}$. Recentemente, Izgi et al. ${ }^{7}$ empregaram planejamento fatorial para otimização de um processo para determinação de mercúrio. Neste caso dezesseis experimentos foram executados, nos

\footnotetext{
*e-mail: zezzi@iqm.unicamp.br
}

quais, o parâmetro mais importante foi aquele relativo ao volume da solução. Em um outro exemplo, Bermejo-Barrera et al. ${ }^{8}$ otimizaram um método de decomposição de organismos marinhos para posterior determinação de metais a baixas concentrações $(\mu \mathrm{g} / \mathrm{kg})$ empregando ETAAS. Em 1997 Hilligsoe e Hansen ${ }^{9}$ combinaram planejamento fatorial e otimização simplex para o desenvolvimento de um sistema de geração de hidretos com posterior determinação de germânio por ETAAS.

Em um outro contexto, a aplicação de modificadores químicos é uma importante ferramenta para estabilizar diferentes analitos a altas temperaturas, aumentar a volatilidade dos concomitantes, o tempo de vida da plataforma de L'vov e a sensibilidade na determinação de elementos com média/alta volatilidade. Além disso, em se tratando de modificadores químicos permanentes, a eliminação de impurezas voláteis presentes em diferentes modificadores ${ }^{10,11}$ e a simplificação operacional (menor número de soluções depositadas no forno), podem ser outras vantagens. Entretanto, a literatura é escassa quando reporta o uso de planejamento fatorial para a otimização das temperaturas de pirólise e atomização ${ }^{12}$, e nenhum trabalho é encontrado quando são envolvidos modificadores químicos permanentes.

Desta forma, o principal objetivo deste trabalho foi o emprego de planejamento fatorial na diminuição do número de experimentos relativos aos estudos das temperaturas de pirólise e atomização em ETAAS, assim como obter informações mais conclusivas sobre os dados obtidos. Para este propósito, planejamento fatorial $2^{2}$ mais ponto central mais planejamento em estrela foi executado para a determinação de $\mathrm{Cd}$ e $\mathrm{Pb}$ (metais com baixas temperaturas de pirólise e atomização), sendo realizados 11 experimentos para cada metal. Foram efetuados, também, planejamentos $2^{2}$ mais ponto central para o Al e Mo (metais com altas temperaturas de pirólise e atomização), sendo executados 7 experimentos para cada metal. Os modificadores químicos permanentes empregados foram $\mathrm{Pd}, \mathrm{W}, \mathrm{Zr}$, Rh, Ir ou suas combinações. 


\section{PARTE EXPERIMENTAL}

\section{Equipamentos}

Na realização dos experimentos foi empregado um espectrômetro de absorção atômica marca Perkin-Elmer (Überlingen, Alemanha), modelo AAnalyst 600 com corretor de fundo por efeito Zeeman, e tubos THGA com plataforma integrada.

Todas as medidas foram baseadas na absorbância integrada, e realizadas a 228,8 $(\mathrm{Cd})$ e 283,3 $\mathrm{nm}(\mathrm{Pb})$, com o emprego de lâmpadas EDL, e a 309,3 (Al) e 313,3 nm (Mo) com o emprego de lâmpadas de cátodo oco.

\section{Materiais, reagentes e soluções}

Foi empregada água destilada e desionizada em todas as etapas experimentais. A solução padrão de $\mathrm{Cd}(1000 \mathrm{mg} / \mathrm{L})$ foi preparada a partir de $\mathrm{CdCl}_{2} \cdot \mathrm{H}_{2} \mathrm{O}$ (Merck, Darmstadt, Alemanha) e a de $\mathrm{Pb}(1000$ $\mathrm{mg} / \mathrm{L}$ ) a partir de $\mathrm{Pb}\left(\mathrm{NO}_{3}\right)_{2}$ (Ecibra, São Paulo, Brasil). As soluções de $\mathrm{Al}$ e Mo (1000 mg/L) foram preparadas a partir de seus óxidos (Vetec, Rio de Janeiro, Brasil).

Os modificadores convencionais foram preparados a partir de $\mathrm{Mg}\left(\mathrm{NO}_{3}\right)_{2}$ (Merck) e $\mathrm{NH}_{4} \mathrm{H}_{2} \mathrm{PO}_{4}$ (Ecibra). Uma solução estoque a $1 \%(\mathrm{~m} / \mathrm{v})$ de $\mathrm{Pd}$ a partir de $\mathrm{Pd}\left(\mathrm{NO}_{3}\right)_{2}$ (Merck) foi empregada como modificador convencional e permanente. Para os modificadores químicos permanentes, $\mathrm{Zr}$ e Ir foram preparados a partir de $\mathrm{ZrO}\left(\mathrm{NO}_{3}\right)_{2}$ e $\mathrm{IrCl}_{3} .3 \mathrm{H}_{2} \mathrm{O}$ (Acros, New Jersey, EUA), $\mathrm{Rh}$ a partir de $\mathrm{RhCl}_{3} .2 \mathrm{H}_{2} \mathrm{O}$ (Sigma, Saint Louis, EUA) e W a partir de $\mathrm{Na}_{2} \mathrm{WO}_{4} \cdot 2 \mathrm{H}_{2} \mathrm{O}$ (Merck). A concentração destes modificadores foi sempre igual a $1 \mathrm{~g} / \mathrm{L}$.

\section{TRATAMENTO DA PLATAFORMA}

Em todos os tratamentos efetuados nas plataformas de L'vov, foi empregado o programa de aquecimento descrito na Tabela 1. Este programa foi composto por apenas quatro etapas, e foi adaptado a partir dos trabalhos de Iwamoto et al. ${ }^{13} \mathrm{e}$ Lima et al. ${ }^{14}$ No primeiro passo, $50 \mu \mathrm{L}$ de uma solução contendo $1 \mathrm{~g} / \mathrm{L}$ do modificador químico permanente foram introduzidos na plataforma e iniciado o programa de aquecimento. $\mathrm{O}$ tratamento foi repetido cinco vezes com o emprego total de $250 \mu \mathrm{g}$ de modificador. Algumas plataformas foram tratadas com dois modificadores, $\mathrm{Zr}$ e $\mathrm{Rh}$ ou $\mathrm{Zr}$ e W. Neste caso, inicialmente a plataforma foi tratada com $250 \mu \mathrm{g} \mathrm{Zr}$ e, depois, com $250 \mu \mathrm{g}$ Rh ou W.
Tabela 1. Programa para tratamento das plataformas de L'vov

\begin{tabular}{ccccc}
\hline Etapa & $\begin{array}{c}\text { Temperatura } \\
\left({ }^{\circ} \mathrm{C}\right)\end{array}$ & $\begin{array}{c}\text { Rampa } \\
(\mathrm{s})\end{array}$ & $\begin{array}{c}\text { Permanência } \\
(\mathrm{s})\end{array}$ & $\begin{array}{c}\text { Fluxo de Ar } \\
(\mathrm{mL} / \mathrm{min})\end{array}$ \\
\hline 1 & 120 & 5 & 25 & 250 \\
2 & 150 & 15 & 10 & 250 \\
3 & 1000 & 20 & 10 & 250 \\
4 & 2000 & 1 & 1 & 250 \\
\hline
\end{tabular}

Deve-se frisar que é comum encontrar na literatura o emprego de combinações entre modificadores que formam carbetos (W) e óxidos $(\mathrm{Rh})^{14,15}$.

\section{Planejamento Fatorial para $\mathrm{Cd}$ e $\mathbf{P b}$}

Para os processos de otimização das temperaturas de pirólise e atomização de $\mathrm{Cd}$ e $\mathrm{Pb}$, foi empregado um planejamento fatorial com 11 experimentos, conforme descritos na Tabela 2. Este fatorial possuiu dois níveis relativos à menor $(-1)$ e maior $(+1)$ temperaturas (ver Tabela 2) e duas variáveis (temperaturas de pirólise e atomização). As temperaturas foram normalizadas entre $-1 \mathrm{e}+1$. Além disso, foram realizados três experimentos no ponto central (temperaturas de pirólise e atomização normalizadas em 0 ) para o cálculo do erro, e 4 experimentos em estrela. Como com o planejamento em estrela se obtêm uma equação de segunda ordem, e as temperaturas de pirólise e atomização são mais baixas, era possível empregar este planejamento, já que o equipamento de atomização empregado permitia o trabalho nessas temperaturas. A configuração dos experimentos pode ser visualizada na Figura 1, onde o quadrado com círculos sólidos nas arestas representa o fatorial $2^{2}$, o círculo aberto no centro representa o ponto central, e o losango com quadrados sólidos nas arestas representa o fatorial em estrela. Depois da execução de todos os experimentos foi obtida uma equação de segunda ordem:

$\mathrm{y}=\beta_{0}+\beta_{1} P+\beta_{2} A+\beta_{3} P^{2}+\beta_{4} A^{2}+\beta_{5} P A$

onde y é a variável dependente (absorbância integrada), e $\beta_{i}$ são os coeficientes para $P$ (temperatura de pirólise) e $A$ (temperatura de atomização), e $P A$ é a interação entre as temperaturas de pirólise e atomização. Para o cálculo dos coeficientes $\left(\beta_{i}\right)$, foi empregado mínimos quadrados:

Tabela 2. Planejamento Fatorial usado para $\mathrm{Cd}$ e $\mathrm{Pb}$

\begin{tabular}{|c|c|c|c|c|c|c|c|c|}
\hline \multirow[b]{3}{*}{ Experimentos } & \multicolumn{4}{|c|}{$\mathrm{Cd}$} & \multicolumn{4}{|c|}{$\mathrm{Pb}$} \\
\hline & \multicolumn{2}{|c|}{ Pirólise } & \multicolumn{2}{|c|}{ Atomização } & \multicolumn{2}{|c|}{ Pirólise } & \multicolumn{2}{|c|}{ Atomização } \\
\hline & $\left({ }^{\circ} \mathrm{C}\right)$ & Nor.* & $\left({ }^{\circ} \mathrm{C}\right)$ & Nor. * & $\left({ }^{\circ} \mathrm{C}\right)$ & Nor. * & $\left({ }^{\circ} \mathrm{C}\right)$ & Nor. * \\
\hline 1 & 400 & -1 & 1400 & -1 & 490 & -1 & 1150 & -1 \\
\hline 2 & 600 & +1 & 1400 & -1 & 910 & +1 & 1150 & -1 \\
\hline 3 & 400 & -1 & 1600 & +1 & 490 & -1 & 1850 & +1 \\
\hline 4 & 600 & +1 & 1600 & +1 & 910 & +1 & 1850 & +1 \\
\hline 5 & 500 & 0 & 1500 & 0 & 700 & 0 & 1500 & 0 \\
\hline 6 & 500 & 0 & 1500 & 0 & 700 & 0 & 1500 & 0 \\
\hline 7 & 500 & 0 & 1500 & 0 & 700 & 0 & 1500 & 0 \\
\hline 8 & 360 & $-\sqrt{2}$ & 1500 & 0 & 400 & $-\sqrt{2}$ & 1500 & 0 \\
\hline 9 & 640 & $+\sqrt{2}$ & 1500 & 0 & 1000 & $+\sqrt{2}$ & 1500 & 0 \\
\hline 10 & 500 & 0 & 1360 & $-\sqrt{2}$ & 700 & 0 & 1000 & $-\sqrt{2}$ \\
\hline 11 & 500 & 0 & 1640 & $+\sqrt{2}$ & 700 & 0 & 2000 & $+\sqrt{2}$ \\
\hline
\end{tabular}

\footnotetext{
* Dados normalizados
} 


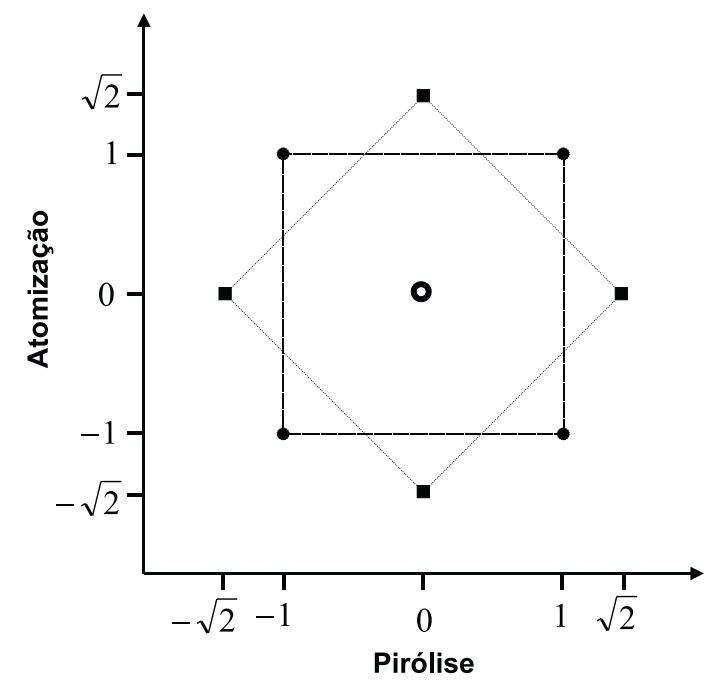

Figura 1. Representação do planejamento fatorial. Quadrado tracejado com círculos sólidos representa o fatorial $2^{2}$, círculo aberto no centro da figura representa o ponto central e o losango tracejado com quadrados sólidos representa o fatorial em estrela

$\beta=\left(X^{t} X\right)^{-1} X^{t} y$

onde $\beta$ é a matriz com coeficientes $\left(\beta_{i}\right), X$ é a matriz com os parâmetros normalizados e y é a resposta experimental.

\section{Planejamento Fatorial para Al e Mo}

No planejamento fatorial para o $\mathrm{Al}$ e o Mo foram executados experimentos $2^{2}$ mais ponto central ( 3 réplicas). Este procedimento foi adotado, pois as temperaturas de pirólise e atomização para esses metais são muito elevadas, sendo que os valores para essas tempera- turas eram maiores do que àqueles permitidos pelo equipamento de absorção atômica, contribuindo para a não execução do planejamento em estrela. Assim, como não foi realizado o planejamento em estrela, não foi obtida uma equação de segunda ordem. Desta forma, a Tabela 3 mostra os experimentos efetuados para os dois metais. Com esses experimentos foi possível obter uma equação de primeiro grau:

$\mathrm{y}=\beta_{0}+\beta_{1} P+\beta_{2} A+\beta_{3} P A$

onde $y$ é a variável dependente (absorbância integrada), e $\beta_{i}$ são os coeficientes para $P$ (temperatura de pirólise) e $A$ (temperatura de atomização), e $P A$ é a interação entre as temperaturas de pirólise e atomização. Para o cálculo dos coeficientes $\left(\beta_{i}\right)$, foi empregado mínimos quadrados, como mostrado na Equação 2.

\section{Condições recomendadas}

Com o propósito de efetuar uma avaliação dos dados obtidos, foram realizados alguns experimentos com as condições recomendadas pelo fabricante ${ }^{16}$, conforme a Tabela 4 . A Tabela 5 mostra o principal programa de aquecimento empregado nos experimentos de otimização. Em todos os experimentos $20 \mu \mathrm{L}$ de uma solução padrão de 2, 50, 30 e $20 \mu \mathrm{g} / \mathrm{L}$ para $\mathrm{Cd}, \mathrm{Pb}, \mathrm{Al}$ e Mo foram empregados, respectivamente.

\section{RESULTADOS E DISCUSSÃO}

\section{Otimização das temperaturas}

Nas otimizações efetuadas para os metais estudados, foram levados em consideração a absorbância integrada e a massa característica $\left(\mathrm{m}_{\mathrm{o}}\right)$. Como parâmetro de comparação nestes estudos, as massas características estabelecidas como ideais para este trabalho, devem ser próximas àquelas do fabricante (Tabela 4).

Tabela 3. Planejamento Fatorial usado para $\mathrm{Al}$ e Mo

\begin{tabular}{|c|c|c|c|c|c|c|c|c|}
\hline \multirow[b]{3}{*}{ Experimentos } & \multicolumn{4}{|c|}{$\mathrm{Al}$} & \multicolumn{4}{|c|}{ Mo } \\
\hline & \multicolumn{2}{|c|}{ Pirólise } & \multicolumn{2}{|c|}{ Atomização } & \multicolumn{2}{|c|}{ Pirólise } & \multicolumn{2}{|c|}{ Atomização } \\
\hline & $\left({ }^{\circ} \mathrm{C}\right)$ & Nor.* & $\left({ }^{\circ} \mathrm{C}\right)$ & Nor. * & $\left({ }^{\circ} \mathrm{C}\right)$ & Nor. * & $\left({ }^{\circ} \mathrm{C}\right)$ & Nor. * \\
\hline 1 & 1100 & -1 & 2200 & -1 & 1400 & -1 & 2350 & -1 \\
\hline 2 & 1300 & +1 & 2200 & -1 & 1600 & +1 & 2350 & -1 \\
\hline 3 & 1100 & -1 & 2400 & +1 & 1400 & -1 & 2550 & +1 \\
\hline 4 & 1300 & +1 & 2400 & +1 & 1600 & +1 & 2550 & +1 \\
\hline 5 & 1200 & 0 & 2300 & 0 & 1500 & 0 & 2450 & 0 \\
\hline 6 & 1200 & 0 & 2300 & 0 & 1500 & 0 & 2450 & 0 \\
\hline 7 & 1200 & 0 & 2300 & 0 & 1500 & 0 & 2450 & 0 \\
\hline
\end{tabular}

* Dados normalizados

Tabela 4. Condições recomendadas para a determinação de $\mathrm{Cd}, \mathrm{Pb}, \mathrm{Al}$ e $\mathrm{Mo}$

\begin{tabular}{|c|c|c|c|c|}
\hline Metal & Pirólise $\left({ }^{\circ} \mathrm{C}\right)$ & Atomização $\left({ }^{\circ} \mathrm{C}\right)$ & $\mathrm{m}_{\mathrm{o}}(\mathrm{pg})$ & Modificador químico* \\
\hline $\mathrm{Cd}$ & 500 & 1500 & 1,3 & $\begin{array}{c}0,005 \mathrm{mg} \text { de } \mathrm{Pd}+0,003 \mathrm{mg} \text { de } \mathrm{Mg}\left(\mathrm{NO}_{3}\right)_{2} \text { ou } \\
0,05 \mathrm{mg} \text { de } \mathrm{NH} \mathrm{HO}+0.003 \mathrm{mg} \text { de } \mathrm{Mg}(\mathrm{NO})\end{array}$ \\
\hline $\mathrm{Pb}$ & $\begin{array}{c}1000 \\
850\end{array}$ & $\begin{array}{l}1900 \\
1600\end{array}$ & $\begin{array}{l}50 \\
30\end{array}$ & $0,005 \mathrm{mg}$ de $\mathrm{Pd}+0,003 \mathrm{mg}$ de $\mathrm{Mg}\left(\mathrm{NO}_{3}\right)_{2}$ \\
\hline $\mathrm{Al}$ & 1200 & 2300 & 31 & $0,015^{2} \mathrm{mg}$ de $\mathrm{Mg}\left(\mathrm{NO}_{3}\right)_{2}$ \\
\hline Mo & 1500 & 2450 & 12 & $0,005 \mathrm{mg}$ de $\mathrm{Pd}+0,003 \mathrm{mg}$ de $\mathrm{Mg}\left(\mathrm{NO}_{3}\right)_{2}$ \\
\hline
\end{tabular}

* volume injetado: $10 \mu \mathrm{L}$ 
Tabela 5. Programa de aquecimento empregado nos experimentos

\begin{tabular}{ccccc}
\hline Etapa & $\begin{array}{c}\text { Temperatura } \\
\left({ }^{\circ} \mathrm{C}\right)\end{array}$ & $\begin{array}{c}\text { Rampa } \\
(\mathrm{s})\end{array}$ & $\begin{array}{c}\text { Permanência } \\
(\mathrm{s})\end{array}$ & $\begin{array}{c}\text { Fluxo de Ar } \\
(\mathrm{mL} / \mathrm{min})\end{array}$ \\
\hline 1 & 110 & 5 & 30 & 250 \\
2 & 130 & 15 & 30 & 250 \\
3 & $*$ & 10 & 20 & 250 \\
4 & $*$ & 0 & $* *$ & 0 \\
5 & $* * *$ & 1 & 3 & 250 \\
\hline
\end{tabular}

*Cd e Pb veja Tabela 2; Al e Mo veja Tabela 3.

**Cd e Al: $3 \mathrm{~s} ; \mathrm{Pb}: 4 \mathrm{~s} ; \mathrm{Mo}: 7 \mathrm{~s}$.

*** $\mathrm{Cd}$ e Pb: $2200{ }^{\circ} \mathrm{C}$; $\mathrm{Al}: 2450{ }^{\circ} \mathrm{C}$; Mo: $2550{ }^{\circ} \mathrm{C}$

\section{Cádmio e Chumbo}

Para os estudos com $\mathrm{Cd}$ e $\mathrm{Pb}$ (Tabela 2) foram empregados apenas modificadores químicos permanentes.

Onze experimentos foram feitos em cada passo da otimização, variando simultaneamente as temperaturas de pirólise e atomização. É importante ressaltar que estes experimentos foram executados em ordem aleatória, para se evitar erros sistemáticos. Depois, os coeficientes para cada modificador químico permanente foram calculados (de acordo com as Equações 1 e 2). Neste contexto, as equações obtidas para cada metal estudado são mostradas na Tabela 6 .

É interessante notar que as Figuras 2 e 3 foram obtidas a partir das equações mostradas na Tabela 6.

\section{Temperaturas de pirólise e atomização para $C d$}

As condições de otimização para o Cd utilizando $\mathrm{Pd}, \mathrm{Zr}$, W e $\mathrm{Zr}+\mathrm{W}$ como modificadores químicos permanentes, apresentaram comportamento similar (Figura $2 \mathrm{a}, \mathrm{c}-\mathrm{e}$ ) relativo à pirólise $\mathrm{e}$ atomização, apesar das diferenças de absorbância integrada observadas para o Pd (Figura 2a) quando comparado com os outros modificadores. Devido às temperaturas obtidas $\left(360\right.$ e $\left.1500^{\circ} \mathrm{C}\right)$ para pirólise e atomização, respectivamente, é possível efetuar a determinação de Cd empregando-se o Pd como modificador químico permanente. Entretanto, devido à baixa temperatura de pirólise obtida o seu uso não é recomendado.

Quando $\mathrm{Zr}$, W e $\mathrm{Zr}+\mathrm{W}$ foram empregados como modificadores permanentes (Figura 2c-e), a temperatura de atomização não foi significante para o modelo proposto pelo planejamento fatorial, porém, a de pirólise foi significante. Depois de aplicar a equação diferencial relativa à pirólise nas equações relacionadas aos modificadores mencionados acima (ver Tabela 6), as temperaturas de pirólise obtidas foram de 470,430 e $430^{\circ} \mathrm{C}$ para $\mathrm{Zr}$, W e $\mathrm{Zr}+\mathrm{W}$, respectivamente. Observando a Figura 2c-e, nota-se que é possível usar temperaturas de pirólise mais altas do que aquelas mencionadas anteriormente (variando de 400 a $550{ }^{\circ} \mathrm{C}$ ). Desta forma, 500 e $1500{ }^{\circ} \mathrm{C}$ foram empregados como temperaturas de pirólise e atomização, respectivamente, e a m obtida foi de 1,4 pg para o $\mathrm{Zr}$ e W (absorbância integrada de 0,13 ), e 1,5 pg para a mistura $\mathrm{Zr}+\mathrm{W}$ (absorbância integrada de 0,11$)$. Neste caso as temperaturas foram maiores que para o $\mathrm{Pd} \mathrm{e}$ as massas características similares às recomendadas (vide Tabela 4).

Para o Rh um comportamento interessante foi observado (Figura 2b), tanto a pirólise como a atomização foram significativas para o planejamento fatorial, já que os coeficientes para $P$ e $A$ (Tabela 6) foram maiores que o erro calculado. Além disso, a partir da Figura $2 \mathrm{~b}$ é possível notar que a temperatura de pirólise empregada pode ser maior que a dos outros modificadores (aproximadamente 640 ${ }^{\circ} \mathrm{C}$ ). A temperatura de atomização foi fixa em $1500{ }^{\circ} \mathrm{C}$. Com as temperaturas fixas em 640 e $1500{ }^{\circ} \mathrm{C}$ para a pirólise e atomização, res- (a)

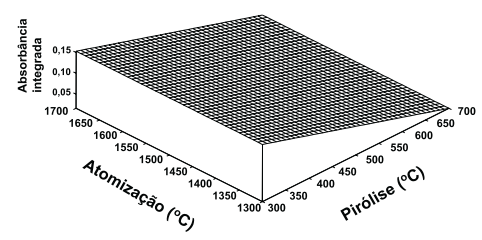

(b)
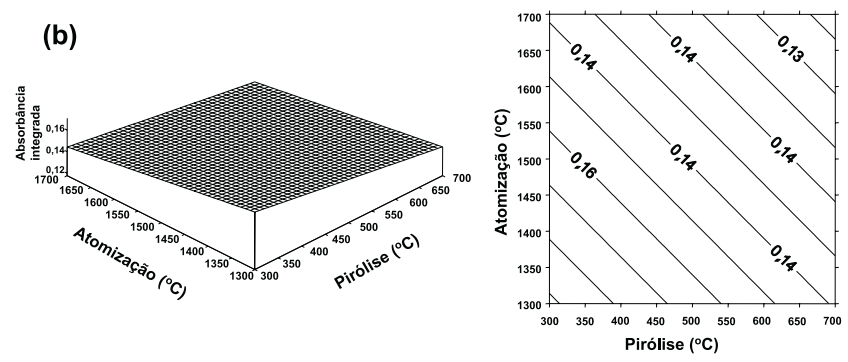

(c)
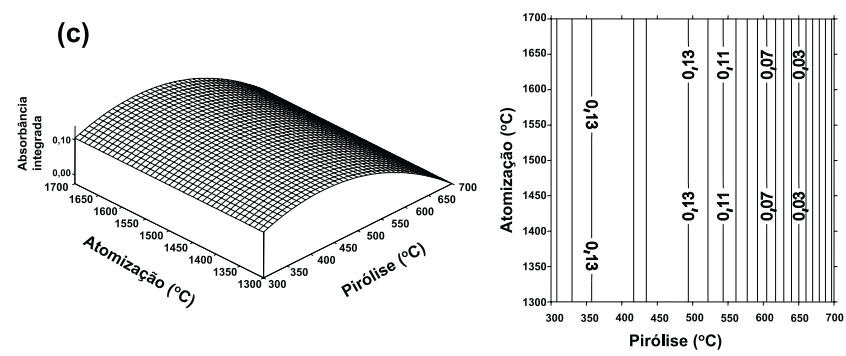

(d)
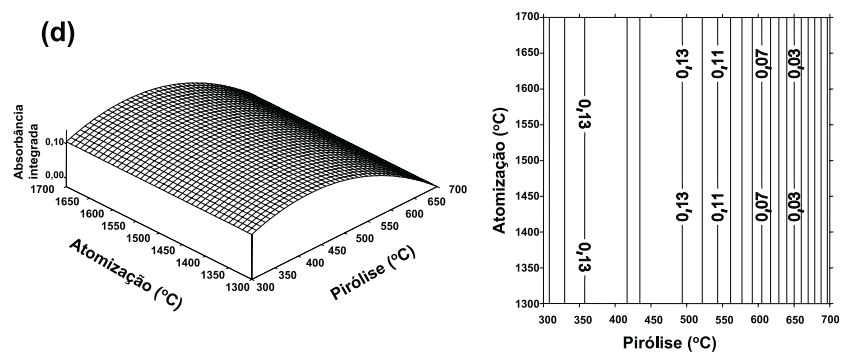

(e)
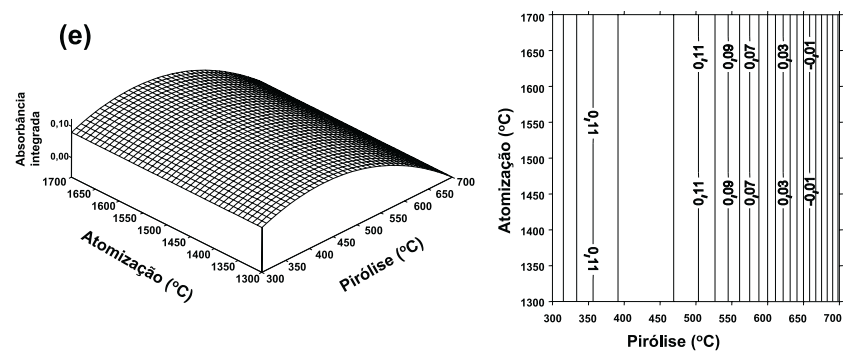

Figura 2. Superfície de resposta (lado esquerdo) e gráficos de contorno (lado direito) obtidos para a otimização das temperaturas de pirólise e atomização para o $\mathrm{Cd}$, onde $\mathrm{Pd}, \mathrm{Rh}, \mathrm{Zr}, \mathrm{W}$ ou $\mathrm{Zr}+\mathrm{W}$ foram empregados como modificadores químicos permanentes em $(a),(b),(c),(d)$ e (e), respectivamente. Os números nos gráficos de contorno referem-se à absorbância integrada 
(a)

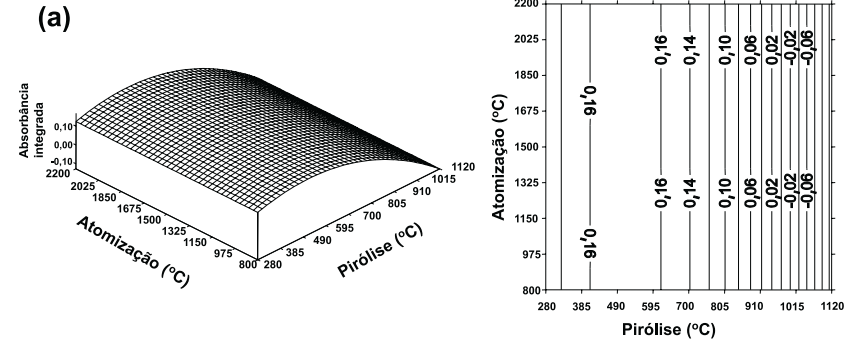

(b)
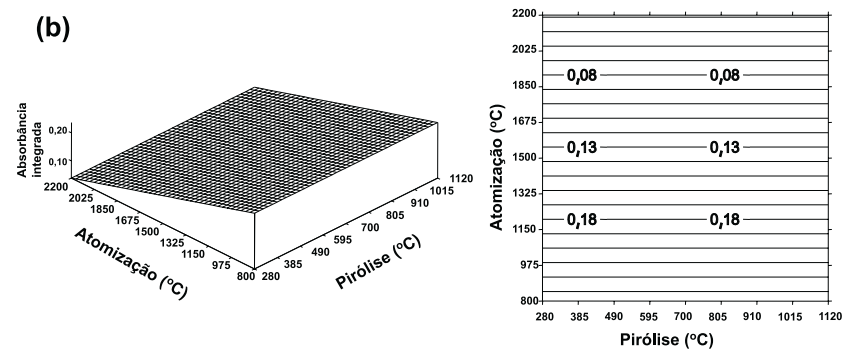

(c)
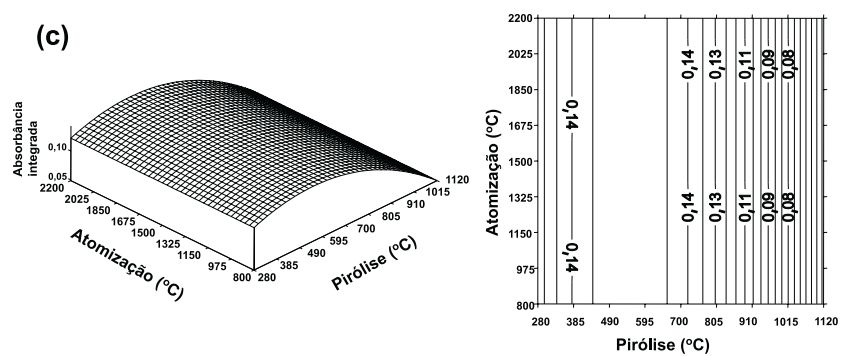

(d)

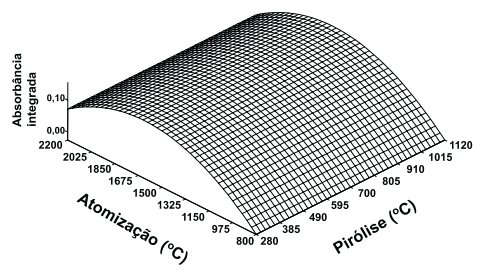

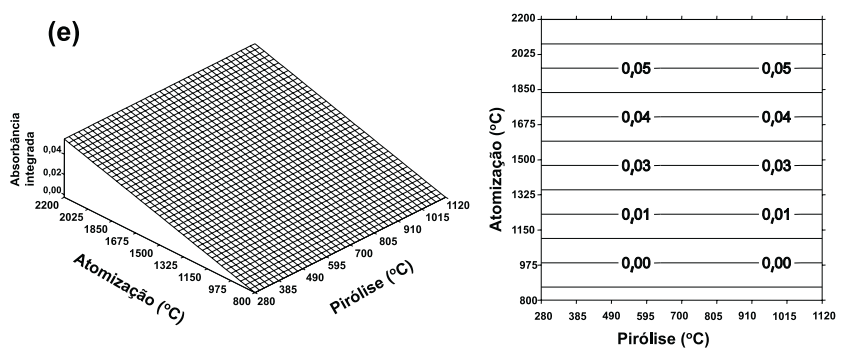
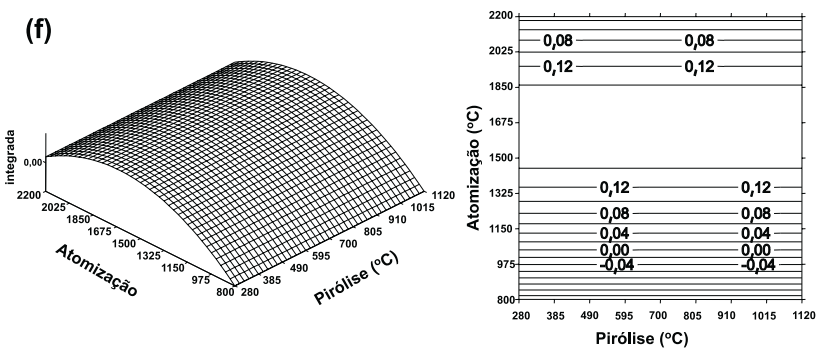

Figura 3. Superfície de resposta (lado esquerdo) e gráficos de contorno (lado direito) obtidos para a otimização das temperaturas de pirólise e atomização para o $\mathrm{Pb}$, onde $\mathrm{Pd}, \mathrm{Rh}, \mathrm{Zr}, \mathrm{W}, \mathrm{Ir}$ ou $\mathrm{Zr}+\mathrm{Rh}$ foram empregados como modificadores químicos permanentes em $(a),(b),(c),(d),(e)$ e $(f)$, respectivamente. Os números nos gráficos de contorno referem-se à absorbância integrada

Tabela 6. Equações obtidas para $\mathrm{Pb}$ e Cd empregando diferentes modificadores químicos permanentes

\begin{tabular}{ccc}
\hline Modificador & $\mathrm{Cd}$ & $\mathrm{Pb}$ \\
\hline $\mathrm{Pd}$ & $y=0,0845-0,0383 P$ & $y=0,1410-0,0628 P-0,0355 P^{2}$ \\
$\mathrm{Rh}$ & $y=0,1443-0,0066 P-0,0067 A$ & $y=0,1376-0,0495 A$ \\
$\mathrm{Zr}$ & $y=0,1222-0,0278 P-0,0530 P^{2}$ & $y=0,1370-0,0184 P-0,0129 P^{2}$ \\
$\mathrm{~W}$ & $y=0,1282-0,0324 P-0,0219 P^{2}$ & $y=0,1513+0,0242 A-0,0319 A^{2}$ \\
$\mathrm{Ir}$ & $y=0,0788$ & $y=0,0226+0,0144 A$ \\
$\mathrm{Zr}+\mathrm{Rh}$ & - & $y=0,1480+0,0461 A-0,0522 A^{2}$ \\
$\mathrm{Zr}+\mathrm{W}$ & $y=0,1138-0,0358 P-0,0256 P^{2}$ & $y=0,1282$ \\
\hline
\end{tabular}

pectivamente, foi obtida uma absorbância integrada de 0,13 e $\mathrm{m}_{\mathrm{o}}=$ 1,4 pg. Os resultados para $m_{0}$ também são concordantes com os do fabricante (Tabela 4).

A superfície de resposta não foi mostrada para o Ir, pois o mesmo produziu uma grande variação de resultados $(0,04-0,09$ absorbância integrada) para a atomização do Cd. Com as temperaturas fixas em 500 e $1500{ }^{\circ} \mathrm{C}$ para a pirólise e atomização, respectivamente, foi obtida uma absorbância integrada de $0,08\left(\mathrm{~m}_{\mathrm{o}}=2,2 \mathrm{pg}\right)$.

\section{Temperaturas de pirólise e atomização para $\mathrm{Pb}$}

O paládio foi o primeiro metal a ser empregado como modificador permanente e a temperatura de atomização obtida não foi significativamente diferente, porque os coeficientes para $A$ (eq. 1) foram menores que o erro calculado a partir do ponto central. Isto pode ser observado a partir da Figura 3a na qual a temperatura de atomização não apresentou variação no tocante a absorbância integrada; entre- 
tanto, uma diferença significativa foi observada para a temperatura de pirólise. Nesse caso, a faixa de temperatura de pirólise que apresentou maior absorbância integrada está entre 400 e $600{ }^{\circ} \mathrm{C}$. Esses valores são menores que o recomendado $\left(850^{\circ} \mathrm{C}\right)^{16}$. Neste caso específico, é possível concluir que o paládio não é um bom modificador químico permanente para a determinação de $\mathrm{Pb}$. Quando a temperatura de pirólise foi de $700{ }^{\circ} \mathrm{C}$ (absorbância integrada igual a 0,14 Figura 3a) uma massa característica $\left(\mathrm{m}_{0}\right)$ de $31 \mathrm{pg}$ foi obtida. Como o efeito da temperatura de atomização não foi significativo, conforme já comentado, a mesma foi adotada como $1600{ }^{\circ} \mathrm{C}^{16}$.

Para o Zr (Figura 3c) foi possível observar o mesmo comportamento que para o $\mathrm{Pd}$. Entretanto, o $\mathrm{Zr}$ estabiliza o $\mathrm{Pb}$ a altas temperaturas. A temperatura máxima obtida após a desnormalização foi de $550{ }^{\circ} \mathrm{C}$ para a temperatura de pirólise. Porém, observando a Figura 3c, lado direito, é possível identificar que a 910 e $1850{ }^{\circ} \mathrm{C}$ para temperaturas de pirólise e atomização, respectivamente, uma $\mathrm{m}_{\mathrm{o}}$ de $37 \mathrm{pg}$ (absorbância integrada igual a 0,11 ) foi obtida. Desta forma, estas temperaturas foram empregadas quando foi usado $\mathrm{Zr}$ como modificador permanente.

De acordo com a Figura 3b,d-f existe uma diferença significativa para a temperatura de atomização enquanto que para a de pirólise isto não foi observado. Quando foi usado Ir como modificador permanente (Figura 3e) a absorbância integrada sempre foi pequena (de 0,01 a 0,05 ), sendo que conclusões sobre esse modificador tornaram-se difíceis. Ao se trabalhar com temperaturas de 910 e $1850{ }^{\circ} \mathrm{C}$ para a pirólise e atomização, respectivamente, uma absorbância integrada de 0,03 foi obtida $\left(m_{\mathrm{o}}\right.$ de $\left.135 \mathrm{pg}\right)$.

Bons resultados em termos de massa característica e temperatura foram obtidos para Rh (Figura 3b), W (Figura 3d) e a mistura $\mathrm{Zr}+\mathrm{Rh}$ (Figura 3f). No caso da determinação de Pb usando Rh como modificador permanente, os termos quadráticos não foram significativos. Entretanto, observando a Figura 3b (lado direito) com temperaturas de pirólise e atomização de 910 e $1850{ }^{\circ} \mathrm{C}$, respectivamente, foi obtida uma absorbância integrada igual a 0,09 ( $\mathrm{m}_{\mathrm{o}}$ de $42 \mathrm{pg}$ ). Os ótimos valores para $\mathrm{W}$ e $\mathrm{Zr}+\mathrm{Rh}$ foram obtidos (Figura $3 \mathrm{~d}$ e $3 \mathrm{f}$ ) com temperaturas de atomização iguais a 1370 e $1340{ }^{\circ} \mathrm{C}$, respectivamente. Observando-se estas figuras, é possível o emprego de temperaturas de atomização mais altas, variando entre 1800 e $2000^{\circ} \mathrm{C}$, com absorbância integrada variando de 0,10 a 0,14 . Entretanto, para se evitar problemas relativo ao tempo de vida do recobrimento, as temperaturas de pirólise e atomização foram fixas em 910 e $1850{ }^{\circ} \mathrm{C}$, respectivamente, com massas características iguais a $34(\mathrm{~W})$ e $36 \mathrm{pg}(\mathrm{Zr}+\mathrm{Rh})$. Deve-se destacar que na maioria das situações, as massas características estão em concordância e, às vezes melhores, que àquelas obtidas pelo fabricante (vide Tabela 4).

Devido ao fato de que não houve variações significativas na absorbância integrada quando $\mathrm{Zr}+\mathrm{W}$ foi usado na determinação de $\mathrm{Pb}$, a superfície de resposta e gráfico de contorno não foram mostrados neste caso. Além disso, um pequeno ganho em sensibilidade foi observado com os resultados obtidos.

\section{Alumínio e Molibdênio}

\section{Temperaturas de pirólise e atomização para Al}

$\mathrm{Na}$ otimização das temperaturas de pirólise e atomização para o $\mathrm{Al}$ foi empregado apenas um planejamento fatorial $2^{2}$ mais ponto central. O planejamento em estrela não foi utilizado, pois as temperaturas de atomização requeridas seriam superiores aos limites operacionais do equipamento $\left(2600{ }^{\circ} \mathrm{C}\right)$. Inicialmente o planejamento fatorial foi efetuado apenas com o modificador químico convencional $(\mathrm{Mg})$, devido ao fato de que as condições recomendadas pelo fabricante não foram atingidas. A equação obtida após o planejamento fatorial foi:

$$
\mathrm{y}=0,0798+0,0162 \mathrm{~A}
$$

A superfície de resposta e o gráfico de contorno são mostrados na Figura 4. Neste caso a temperatura de pirólise não foi significativa, e é possível identificar que bons resultados podem ser obtidos com temperaturas de atomização maiores que $2400{ }^{\circ} \mathrm{C}$. Entretanto, temperaturas superiores a essa não foram testadas para se evitar um rápido desgaste da plataforma de L'vov. Neste caso, as temperaturas de pirólise e atomização foram fixas em $1100{ }^{\circ} \mathrm{C}$ e $2400{ }^{\circ} \mathrm{C}$, respectivamente.
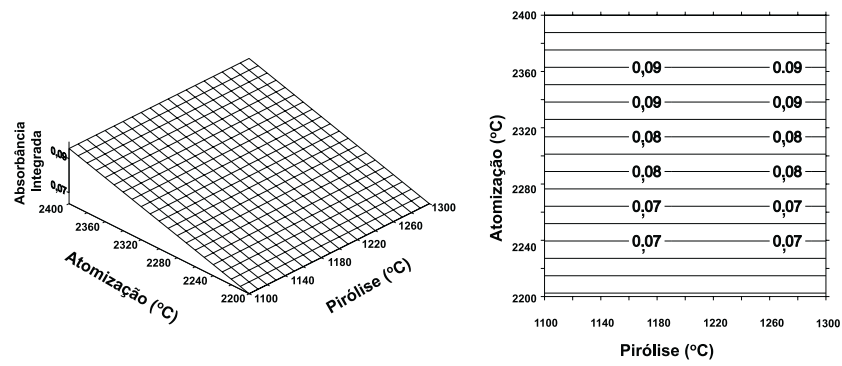

Figura 4. Superfície de resposta (lado esquerdo) e gráficos de contorno (lado direito) obtidos para a otimização das temperaturas de pirólise e atomização para o Al. Os números nos gráficos de contorno referem-se à absorbância integrada

Nos estudos realizados para o $\mathrm{Al}$ não foram efetuados testes com o Pd como modificador químico permanente, devido aos resultados inadequados obtidos para $\mathrm{Cd}$ e $\mathrm{Pb}$. Após definidas as temperaturas de pirólise e atomização, efetuaram-se alguns testes com $\mathrm{Rh}, \mathrm{W}, \mathrm{Ir}, \mathrm{Zr}$, Mo e $\mathrm{Zr}+\mathrm{W}$. Nos testes com o Al, ainda foi feita a tentativa em se empregar o Mo como modificador químico permanente. Os resultados obtidos foram iguais a 30,5, 27,1, 55,2 e 26,4pg para o W, Zr, Mo e $\mathrm{Zr}+\mathrm{W}$, respectivamente. No caso do Rh e do Ir não foi possível obter conclusões efetivas, pois as absorbâncias integradas obtidas foram pequenas $(0,0016$ a 0,0033$)$. Os melhores resultados no tocante ao $\mathrm{Al}$, em comparação ao fabricante, foram aqueles obtidos empregando $\mathrm{Zr}$, W ou a mescla desses dois modificadores (Tabela 4).

\section{Temperaturas de pirólise e atomização para Mo}

Como no caso do Al, as condições recomendadas não produziram bons resultados para o Mo, e somente um planejamento fatorial foi feito. A equação obtida foi:

$$
y=0,0665+0,0174 A
$$

Neste caso, o planejamento em estrela também não foi efetuado, devido às altas temperaturas de pirólise e atomização requeridas. Assim, as temperaturas de pirólise e atomização foram fixas em 1400 e $2500{ }^{\circ} \mathrm{C}$, respectivamente. A superfície de resposta e o gráfico de contorno são mostrados na Figura 5.

Definidas as temperaturas de pirólise e atomização, os modificadores químicos permanentes foram testados. Os resultados obtidos foram ruins, sendo difícil atribuir alguma tendência ou comportamento. O Pd não foi testado, devido aos maus resultados anteriormente obtidos. Além disso, notou-se que a determinação de Mo é possível, inclusive, em ausência de modificadores. Desta forma, a massa característica na ausência de modificadores foi igual a 22,9 pg e na presença de Rh igual a 31,5 pg.

\section{Considerações Gerais}

As temperaturas de pirólise e atomização para a determinação de Cd usando $\mathrm{Pd}, \mathrm{Rh}, \mathrm{Zr}, \mathrm{W}, \mathrm{Zr}+\mathrm{W}$ e $\mathrm{Pb}$ usando $\mathrm{Pd}, \mathrm{Rh}, \mathrm{Zr}$, W, Ir, 


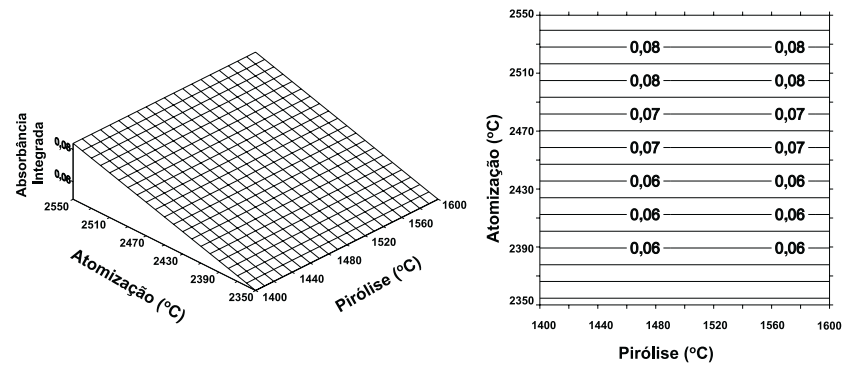

Figura 5. Superfície de resposta (lado esquerdo) e gráficos de contorno (lado direito) obtidos para a otimização das temperaturas de pirólise e atomização para o Mo. Os números nos gráficos de contorno referem-se à absorbância integrada
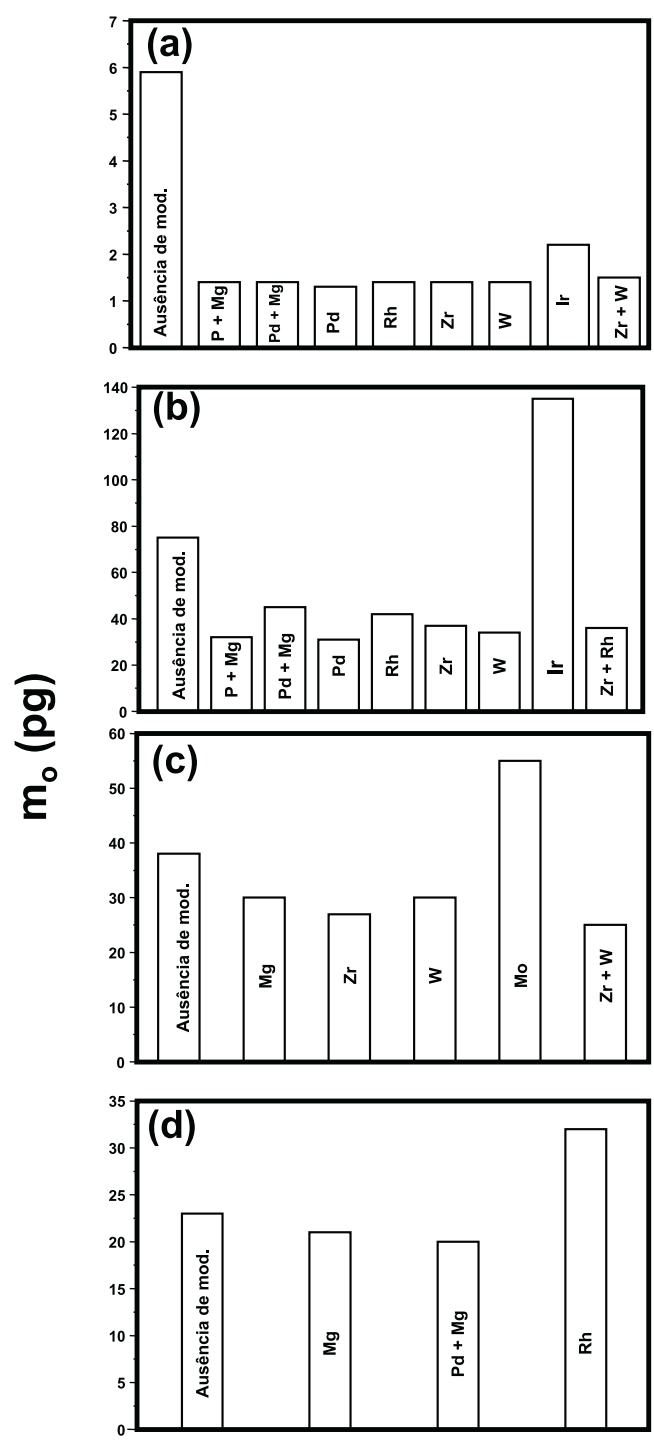

Figura 6. Resultados obtidos em todos os experimentos, onde as Figuras $6 a, 6 b, 6 c$ e $6 d$ representam as massas características obtidas para $\mathrm{Cd}, \mathrm{Pb}$, Al e Mo, respectivamente. A primeira coluna (sem modificador) corresponde à determinação do metal na ausência de modificador permanente ou convencional. As outras colunas, $P+M g, P d+M g, M g$, correspondem à modificação convencional (recomendada pelo fabricante) e as colunas marcadas com $P d, R h, Z r, W, I r, Z r+R h, Z r+W$ e Mo correspondem ao uso de modificadores químicos permanentes
$\mathrm{Zr}+\mathrm{Rh}$ como modificadores químicos permanentes, foram obtidas observando as superfícies de resposta e os gráficos de contorno. $\mathrm{O}$ cálculo matemático para encontrar as melhores temperaturas de pirólise e atomização não foi efetuado simultaneamente para as duas temperaturas, pois os termos quadráticos $\left(P^{2}\right.$ e $\left.A^{2}\right)$ não foram significativos (ver Tabela 6). Neste sentido, apresenta-se na Figura 6, todos os resultados obtidos para $\mathrm{m}_{\mathrm{o}}$ efetuados após o processo de otimização com planejamento fatorial.

O irídio não apresentou resultados satisfatórios para nenhum metal estudado. Este fato pode ser decorrente de diversos fatores, tais como: tipo de recobrimento efetuado, tipo de analito estudado e tipo do sal empregado no preparo do modificador. Na literatura identifica-se uma tendência em se empregar o Ir como modificador químico permanente na determinação de metais geradores de hidretos. Esta afirmação pode ser confirmada com os trabalhos de Shuttler $e t$ al. ${ }^{17}$ e Tsalev et al. ${ }^{18}$.

É importante também frisar que com o emprego de planejamento fatorial é possível obter um maior número de informações analíticas. Esta afirmação é ilustrada através da Figura 7, que representa alguns experimentos executados para a determinação de $\mathrm{Cd}$ com o emprego do $\mathrm{Zr}$ como modificador químico permanente. Os círculos sólidos representam o planejamento fatorial mais ponto central mais estrela, e os quadrados abertos representam os experimentos efetuados em um procedimento univariado. Esta figura mostra que com somente $11 \mathrm{ex}-$ perimentos a determinação de Cd pode ser efetuada a partir de 360 a $600{ }^{\circ} \mathrm{C}$ para pirólise e de 1300 a $1700{ }^{\circ} \mathrm{C}$ para atomização.

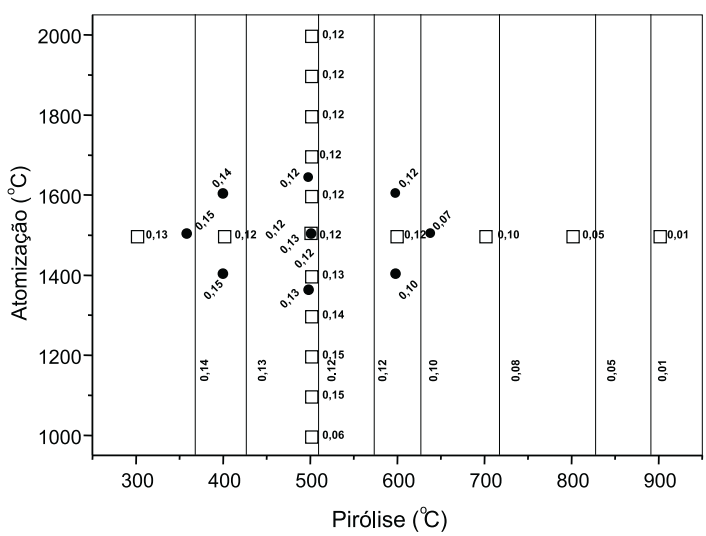

Figura 7. Otimização das temperaturas de pirólise e atomização para $\mathrm{Cd}$ com $\mathrm{Zr}$ como modificador químico permanente. Os círculos sólidos representam os 11 experimentos fatoriais, e os quadrados abertos representam os 17 experimentos executados em uma forma univariada

Outro ponto a ser destacado é o menor número de experimentos obtidos com o planejamento fatorial, em comparação com o método tradicional (univariado). Foram realizados no máximo 11 experimentos na otimização das temperaturas de pirólise e atomização, contra 17-25 apresentados na literatura ${ }^{18-20}$.

\section{CONCLUSÃO}

Este trabalho aponta para alternativas na otimização de curvas de pirólise e atomização, com uma diminuição efetiva do número de experimentos e, ainda, mantendo-se ou melhorando-se as massas características relativas àquelas determinadas pelo fabricante. Deve-se ressaltar que os programas computacionais para a execução de planejamento fatorial são de fácil aquisição, sendo disponíveis na internet. Como exemplo, podemos citar: http://www.chemkeys.com/ bra/index.htm 
Finalmente, com as superfícies de resposta obtidas, diferentes temperaturas para o mesmo analito podem ser usadas em diferentes matrizes a serem avaliadas. Como proposta de possíveis investigações futuras, seria a utilização do planejamento fatorial no sentido de melhora na seletividade dos métodos, possibilitando a escolha de temperaturas de trabalho, nas quais os resultados obtidos, mesmo em presença dos concomitantes, fossem aceitáveis.

\section{AGRADECIMENTOS}

Os autores agradecem à Fundação de Amparo a Pesquisa do Estado de São Paulo (FAPESP processos $n^{\text {os }}$ 98/16548-3 e 99/002595) e ao Conselho Nacional de Desenvolvimento Científico e Tecnológico (CNPq e PADCT-III) pelas bolsas e suporte financeiro.

\section{REFERÊNCIAS}

1. Valcárcel, M.; Trends Anal. Chem. 1997, 16, 124.

2. Beebe, K. R.; Kowalski, B. R.; Anal. Chem. 1987, 59, 1017A.

3. Box, G. E. P.; Hunter, W. G.; Hunter, J. S.; Statistics for experiments. An introduction to design, data analysis and model building; Wiley: New York, 1978.

4. Barros Neto, B.; Scarminio, I. S.; Bruns, R. E., Planejamento e Otimização de Experimentos; Editora da Unicamp: Campinas, 1996.
5. Benzo, Z.; Montero, T.; Quintal, M.; Sierraalta, A.; Ruette, F.; J. Anal. At. Spectrom. 1996, 11, 445.

6. Wegscheider, W.; Knapp G.; Spitzy, H.; Fresenius' J. Anal. Chem. 1977, $283,9$.

7. Izgi, B.; Demir, C.; Güçer, S.; Spectrochim. Acta, Part B 2000, 55, 971.

8. Bermejo-Barrera, P.; Moreda-Piñeiro, A.; Muñiz-Naveiro, O.; GómezFernández, A. M. J.; Bermejo-Barrera, A.; Spectrochim. Acta, Part B 2000, $55,1351$.

9. Hilligsoe, B.; Hansen, E. H.; Fresenius' J. Anal. Chem. 1997, 358, 775.

10. Tsalev, D. L.; Slaveykova, V. I.; Lampugnani, L.; D’ulivo, A; Georgieva, R.; Spectrochim. Acta, Part B 2000, 55, 473.

11. Volynsky, A. B.; Sedykh, E. M.; J. Anal. At. Spectrom. 1989, 4, 71.

12. Legret, M.; Divet, L.; Analusis 1988, 16, 97.

13. Iwamoto, E.; Shimazu, H.; Yokota, K.; Kumamaru, T.; J. Anal. At. Spectrom. 1992, 7, 421.

14. Lima, E. C.; Krug, F. J.; Jackson, K. W.; Spectrochim. Acta, Part B 1998, 53, 1791.

15. Lima, E. C.; Barbosa Jr., F.; Krug, F. J.; Guaita, U.; J. Anal. At. Spectrom. 1999, 14, 1601.

16. The THGA Graphite Furnace: Techniques and Recommended Conditions, Perkin-Elmer: Überlingen, 1995.

17. Shuttler, I. L.; Feuerstein, M.; Schlemmer, G.; J. Anal. At. Spectrom. 1992, 7, 1299.

18. Tsalev, D. L.; D’ulivo, A.; Lampugnani, L.; Marco, M.; Zamboni, R.; J. Anal. At. Spectrom. 1995, 10, 1003.

19. Pyrzynska, K.; Spectrochim. Acta, Part B 1995, 50, 1595.

20. Tsalev, D. L.; D’ulivo, A.; Lampugnani, L.; Marco, M.; Zamboni, R.; J. Anal. At. Spectrom. 1996, 11, 979. 